\title{
Transporte inter-hospitalar de pacientes críticos: opiniões dos profissionais de saúde que realizam o transporte
}

\author{
Aldaíza Ferreira Antunes Fortes, M.Sc.*, Ana Maria Nassar Cintra Soane, M.Sc.**, \\ Sheila Aparecida dos Santos***
}

*Enfermeira, Docente da disciplina Estágio Supervisionado, do Curso de Graduação em Enfermagem, da Escola de Enfermagem Wenceslau Braz (EEWB), Responsável pelo Setor de Pesquisa e Estudos Acadêmicos da EEWB, ItajubálMG, ${ }^{* *}$ Coordenadora do Departamento de Ensino e Pesquisa da EEWB, ItajubálMG, ${ }^{* * *}$ Enfermeira, Acadêmica do curso de Especialização de Enfermagem em Urgência e Emergência e Terapia Intensiva da Escola de Enfermagem Wenceslau Braz (EEWB), ItajubälMG

\section{Resumo}

Estudo descritivo-exploratório e transversal, de abordagem qualitativa, desenvolvido com o objetivo de conhecer as opiniôes dos profissionais de saúde (médicos, enfermeiros, técnicos e auxiliares de enfermagem) sobre o transporte interhospitalar realizado dos pacientes críticos da cidade de Paraisópolis, Sul de Minas Gerais. A amostra foi constituída por todos os enfermeiros, técnicos e auxiliares de enfermagem e médicos plantonistas do Hospital e Maternidade Frei Caetano, que acompanhavam o transporte de pacientes críticos e aceitaram participar do estudo. A amostragem foi intencional. A coleta de dados foi realizada, após aprovação do estudo pelo Comitê de Ética em Pesquisa, utilizando um roteiro de entrevista semiestruturada em duas etapas: a primeira referente às características pessoais da população estudada, e a segunda composta por três questóes abertas inerentes ao objetivo da pesquisa. Os dados foram analisados e interpretados por meio da análise de conteúdo de Bardin. Constatou-se que o transporte inter-hospitalar é uma prática cotidiana que merece melhor atenção, visto que os funcionários se mostram interessados em prestar assistência ao paciente e reconhecem a complexidade do tema, admitindo, porém, que lhes faltam recursos humanos e materiais, assim como capacitaçáo, para atuar com segurança e conhecimento.

Palavras-chave: transporte inter-hospitalar, paciente crítico, equipe de saúde.

\section{Abstract}

Inter-hospital transportation of critical ill patients: health professional views who perform the transportation

This study was a descriptive exploratory and transversal with a qualitative approach developed with the main objective to know the opinions of health professionals (doctors, nurses, technicians and nurse assistants), about the transportation inter-hospitals carried out with patients at risk of Paraisopolis City, South of Minas Gerais. All nurses, technicians, nurse assistants and doctors of the Maternity Frei Caetano participated. The sampling was intentional. Data collecting used an semi-structured interview in two stages: First one refers to the personal characteristics of the studied population, and the 
second consists in three open questions related to the objective of the study. Data was analyzed and interpreted through Bardin content analyses. It was made clear that inter-hospitals transportation is a daily practice that deserves more attention once that the staff showed themselves interested in assisting the patient and they also realized the complexity involving this matter. They admit, however, that there is a shortage of human and material resources and even further skills for them to work with safety and knowledge.

Key-words: inter-hospital transportation, patient at risk, health team.

\section{Resumen}

\section{Transporte interhospitalario de pacientes críticos: opiniones de profesionales de salud que realizan el transporte}

Estudio descriptivo de enfoque cualitativo transversal y exploratorio, desarrollado con el objetivo de conocer las opiniones de profesionales de la salud (médicos, enfermeras, técnicos y auxiliares de enfermería) sobre el transporte interhospitalario realizado de los pacientes críticos de la ciudad de Paraisópolis, Sur de Minas Gerais. La muestra fue constituida por todos los enfermeros, técnicos y auxiliares de enfermería y médicos de guardia del Hospital y Maternidad Frei Caetano, que acompañaban el transporte de pacientes críticos y aceptaron hacer parte del estudio. El muestreo fue intencional. La recopilación de datos se llevó a cabo después de la aprobación del estudio por el Comité de Ética en la investigación mediante un guión de entrevista estructurada formada en dos pasos: la primera relativa a las características personales de la población de estudio y el segundo, compuesto por tres preguntas abiertas inherentes en el objetivo de la encuesta. Los datos fueron analizados e interpretados por análisis de contenido de Bardin. Se observó que el transporte interhospitalario es una práctica cotidiana que merece una mejor atención, ya que los empleados se interesan en prestar asistencia al paciente y reconocen la complejidad de la cuestión, admitiendo, sin embargo, que carece de recursos humanos y materiales, así como capacitación para servir con seguridad y conocimiento.

Palabras-clave: transporte interhospitalario, pacientes críticos.

\section{Introdução}

O transporte inter-hospitalar é necessário quando o hospital de origem não tem recursos para dar continuidade ao tratamento. Ao se optar pelo transporte, deve-se considerar o risco/ benefício, a avaliação do quadro clínico e a estabilização do paciente devem ser feitas antes do início do transporte, e este deve ser efetuado por equipe treinada contando com equipamentos e materiais de qualidade.

Considerando a complexidade do transporte de pacientes críticos, observou-se que o transporte inter-hospitalar é uma prática frequente e, na maioria das vezes, negligenciado pelos profissionais que o executam, que desconhecem dos riscos e das complicações que podem ocorrer em um transporte mal planejado.

As intercorrências passíveis de ocorrer durante o transporte podem se associar às alteraçóes fisiológicas, equipamentos e à equipe de transporte. As intercorrências clínicas são bastante frequentes como: alteraçôes hemodinâmicas, bradicardia e hipóxia, problemas com a pressão arterial e a frequência respiratória, temperatura corporal, além de alterações dos gases sanguíneos [1].
A evolução tecnológica permitiu o avanço dos suportes ventilatórios, entre outros, incrementando o transporte de doentes graves para locais onde o seu tratamento pode ser otimizado, da mesma forma nos últimos anos tem-se verificado um aumento progressivo de unidades com especificidade no tratamento de certas patologias, o que por sua vez teve também como consequência um aumento das transferências de doentes críticos [2].

Este estudo visa conhecer a realidade do transporte inter-hospitalar de uma cidade de pequeno porte, podendo assim propor mudanças no atendimento, favorecendo aos profissionais conhecimentos necessários para efetuar um transporte com eficácia e conscientizando as instituiçôes da necessidade de meios de transporte equipados, para que a equipe exerça seu trabalho com qualidade e segurança. Além disso, contribuir com a profissão da enfermagem com aquisição de conhecimentos, proporcionando o crescimento da profissão.

Diante do exposto este estudo tem como objetivo conhecer as opinióes dos médicos, enfermeiros, técnicos e auxiliares de enfermagem do Hospital Frei Caetano, da cidade de Paraisópolis, Sul de Minas Gerais, acerca do transporte inter-hospitalar dos pacientes críticos. 


\section{Material e métodos}

O presente estudo se define como descritivo, exploratório e transversal, e foi desenvolvido a partir da perspectiva da abordagem qualitativa no Hospital e Maternidade Frei Caetano, em Paraisópolis, Sul de Minas Gerais. Os sujeitos da pesquisa foram 24, sendo duas (2) enfermeiras, três (3) médicos, dezessete (17) técnicos de enfermagem e duas (2) auxiliares de enfermagem.

Os critérios para inclusão dos participantes do estudo foram os seguintes: ser enfermeiro, técnico, auxiliar de enfermagem ou médico plantonista do Hospital e Maternidade Frei Caetano, da cidade de Paraisópolis/MG; ter acompanhado mais de um transporte inter-hospitalar de pacientes críticos; concordar em participar da pesquisa e assinar o termo de consentimento livre e esclarecido. Os critérios de exclusão foram aqueles que não se adequaram nos critérios de inclusão.

$\mathrm{O}$ instrumento utilizado para coleta de dados foi um roteiro de entrevista semiestruturada que consta de duas etapas: a primeira se refere às características pessoais da populaçáo estudada, e a segunda é composta por três questóes abertas inerentes ao objetivo da pesquisa. A própria pesquisadora aplicou os instrumentos. As respostas foram gravadas e a seguir transcritas conforme os critérios metodológicos, permitindo a fidedignidade das informaçôes colhidas. Em seguida, a gravação foi excluída.

A coleta de informaçóes se iniciou após aprovação do Comitê de Ética em Pesquisa da EEWB e liberação dos responsáveis técnicos da instituição inerente ao estudo. Esta foi realizada em julho de 2011. Antes da coleta das informaçóes foi verificado, também, se os respondentes atendiam aos critérios de inclusão, já mencionados anteriormente, e realizou-se a assinatura do Termo de Consentimento Livre e Esclarecido (TCLE).

O pré-teste envolveu três profissionais da instituição, os quais fizeram parte da amostra, visto que não houve necessidade de alterar o instrumento.

Como método de análise dos dados foi utilizado a análise de conteúdo, que abrange três etapas: a pré-análise, o tratamento dos resultado obtidos e a interpretação [3].

Os aspectos éticos do presente estudo obedeceram à Resoluçáo 196/96 do Conselho Nacional de Saúde, concernentes à realização de pesquisas com seres humanos. Cada profissional foi identificado pela codificaçáo $\mathrm{P}_{1}, \mathrm{P}_{2}, \mathrm{P}_{3}$, proveniente da palavra profissional e do número ordinal sequencial de acordo com o número de participantes.

\section{Resultados e discussão}

Em relação às características sociais 17 são do gênero feminino e 6 do masculino. A faixa etária varia de 20 a 64 anos e o tempo de atuação profissional de dois (2) meses a 35 anos. Quanto à especializaçáo, 18 não possuem especializaçáo e os demais apresentam especialização na área de clínica médica, resgate/socorrista, pneumologia, medicina da família e comunidade e, um (01) está cursando faculdade de enfermagem. Dos participantes 12 trabalham em outra instituiçáo, e todos fazem transporte de pacientes críticos.

Quanto às questóes abertas inerentes aos objetivos do estudo do tema "Como é realizado o transporte inter - hospitalar de pacientes críticos" surgiram as seguintes categorias:

\section{Categoria 1 - Ambulância da instituiçáo com acompanhamento do médico, enfermeira ou técnico de enfermagem e um familiar}

A ambulância é o meio de transporte indicado para áreas urbanas, em cidades de pequeno, médio e grande porte, ou para transferências intermunicipais, onde as estradas permitem que essas unidades de transporte se desloquem com segurança e no intervalo de tempo desejável ao atendimento de cada caso [4].

Conforme $20,8 \%$ dos entrevistados o transporte do paciente crítico é acompanhado pelo médico e o técnico de enfermagem. $8,3 \%$ apontam para a presença do familiar no transporte.

\footnotetext{
"Vai de ambulância junto com o médico, o técnico de enfermagem e um familiar." (P1)

"Vai o médico e um técnico de enfermagem." (P3)
}

Dos participantes do estudo, 41,6\% consideram o enfermeiro como parte da equipe que realiza o transporte. A atuaçáo do enfermeiro está relacionada à assistência direta ao paciente grave sob risco de morte. $\mathrm{O}$ profissional que atua em situação de urgência deve possuir formação e experiência profissional, extrema competência, 
habilidade, capacidade física, capacidade de lidar com o estresse, capacidade de tomar decisóes rapidamente, de definir prioridades e saber trabalhar em equipe [5]

A atuaçáo do enfermeiro junto ao paciente crítico vem constantemente se ampliando e tornando-se imprescindível e definitiva.

Conforme a resolução COFEN no 379/2011, que entrou em vigor a partir do dia 01 de janeiro de 2012 a assistência de Enfermagem em qualquer tipo de unidade móvel (terrestre, aérea ou marítima) destinada ao Atendimento Pré-Hospitalar e Inter-Hospitalar, em situaçóes de risco conhecido ou desconhecido, somente deve ser desenvolvida na presença do Enfermeiro [6].

"Então, vai um médico, a enfermeira padrão e um técnico de enfermagem.” (P7)

"Vai o técnico, uma enfermeira padrão, mais o médico, né." (P9)

Para 8,3\% dos entrevistados o transporte inter- hospitalar de pacientes críticos é realizado sem a presença do profissional médico.

"Na nossa unidade tem escala de remoção, só que não são todos os dias que tem médico na cobertura, às vezes falta médico. Tem vezes que sai daqui com paciente sem médico... só com a enfermagem." (P18)

“... daí quando consegue a vaga, não tem médico que queira ir junto, isso que é o complicado." (P 24)

4,1\% dos profissionais participantes desta pesquisa relatam que o médico é quem avalia a necessidade de acompanhar o paciente.

"O médico avalia se ele vai acompanhar ou não vai acompanhar. Se ele não acompanha, ele assina no prontuário que ele avaliou o paciente e liberou para ser acompanhado só com a enfermagem.” (P3)

O transporte do paciente crítico deve sempre ser acompanhado por uma equipe multiprofissional, capaz de oferecer suporte com qualidade durante o trajeto.

\section{Categoria 2 - Pelo sistema SUS Fácil}

$37,5 \%$ dos entrevistados referem que os transportes de pacientes críticos são realizados pelo sistema SUS Fácil. O SUS Fácil é um sistema informatizado de regulação assistencial criado, em 2006, com o intuito de agilizar a troca de informaçóes entre as unidades administrativas e executoras dos serviços de saúde de Minas Gerais, garantindo assim, melhorias constantes no acesso e atendimento prestado a populaçáo [7].

Podemos notar uma insatisfação dos entrevistados em relação ao sistema do SUS Fácil.

"E é terrível pra transferir o paciente do SUS, agora tem o SUS Fácil, às vezes tem paciente que fica até uma semana no pronto socorro esperando transferência...” (P15)

\section{Categoria 3 - Com todo o equipamento neces- sário}

Durante o transporte devem ser mantidas as mesmas medicaçôes, padróes respiratórios e monitorização que o paciente estava recebendo anteriormente. A monitorização mínima necessária consta de: eletrocardiograma e oximetria de pulso contínuas com documentação periódica, medida intermitente e documentação de pressão arterial e freqüência respiratória. A temperatura deve ser avaliada periodicamente, especialmente em crianças e recém-nascidos, pois tanto hiper como hipotermia podem ser prejudiciais [8].

Podemos perceber na fala de $29,1 \%$ dos entrevistados a preocupação com a monitorização.

"Quando o paciente é grave a gente leva o monitor cardíaco, leva monitorizado, leva aspirador." (P15)

41,6\% dos entrevistados citam a preocupação com a via aérea.

"Os pacientes graves saem daqui com a enfermagem, com o médico, vai motorizado, intubado." (P4)

\section{Categoria 4 - Pela UTI móvel}

A prefeitura municipal de Paraisópolis/MG possui um convênio com o Consórcio Intermuni- 
cipal de Saúde (CISMAS), cuja sede fica na cidade de Itajubá, onde é oferecido o serviço de remoção, contando com uma UTI móvel, com profissional médico e técnico de enfermagem. Quando há necessidade de transportar pacientes críticos que necessitam de uma UTI móvel, o transporte é realizado por esse serviço. $\mathrm{O}$ que podemos perceber na fala de $20,8 \%$ dos entrevistados.

"Os pacientes graves saem daqui na ambulância do CISMAS, que é a ambulância UTI que vem, e ela vem já com o médico, a equipe de enfermagem tudo certinho pra levar o paciente pro hospital que for regulamentado pelo SUS Fácil.” (P5)

\section{Categoria 5 - Fora das condiçóes adequadas de transporte}

Para 4,1 \% dos entrevistados o transporte inter-hospitalar de pacientes críticos é feito fora das condiçóes de transporte.

\footnotetext{
"Na minha opinião, pacientes graves não devem ser transportados, a não ser que estejam estáveis hemodinamicamente. Aqui a unidade de emergência tem condiçóes de estabilizar este paciente... inclusive a história que eu escuto aqui do hospital é justamente o transporte de pacientes críticos fora de condição de transporte, sem condição de ser transportado e chega nos outros hospitais em condiçôes mais críticas ainda." (P6)
}

Nota-se na fala do entrevistado a preocupação com o transporte do paciente instável, visto que o transporte deve ser realizado para melhorar as condiçôes do paciente e não para agravar ainda mais seu estado de saúde, para tanto ao se optar pelo transporte devem ser avaliados os benefícios para o paciente.

A análise das respostas à pergunta "Fale sobre os recursos humanos e materiais que possuem" foi dividida em duas categorias: recursos materiais e recursos humanos utilizados no transporte de pacientes críticos.

Em relação aos recursos materiais, 16,6\% dos entrevistados dizem que os recursos materiais que possuem para a realizaçáo do transporte são os medicamentos; $58,3 \%$ relatam que possuem somente equipamentos para monitorização e ventilação;
$20,8 \%$ falam que possuem recurso material suficiente para o mínimo; $4,1 \%$ dizem possuir material de semi UTI; para 16,6\% os recursos materiais são insuficientes e $8,3 \%$ referem falha no funcionamento do equipamento.

"Nós temos na ambulância, é... uma caixa de urgência, com medicação necessária, se caso dá uma parada...” (P7)

Uma caixa de medicaçóes deve acompanhar o transporte do paciente crítico, e as principais são: adrenalina, lidocaína, atropina, bicarbonato de sódio, adenosina, aminofilina, cloreto de cálcio, dexametasona, digoxina, dopamina, furosemida, heparina, manitol, magnésio, naloxone, nitroglicerina, nitroprussiato, fenitoína, cloreto de potássio, procainamida, propranolol e verapamil. Deve-se antecipar a necessidade de medicaçóes prescritas para o paciente, como é o caso da sedação e bloqueadores neuromusculares [9].

\footnotetext{
"Nós temos o oximetro e o monitor cardíaco é esses aparelhos que a gente tem disponível para levar na ambulância." (P7)
}

Existem monitores modulares de transporte, que permitem o acompanhamento contínuo de vários parâmetros vitais: eletrocardiografia, oximetria de pulso, capnografia, pressão arterial não invasiva e módulos de pressáo invasiva. $\mathrm{Na}$ ausência de um monitor múltiplos parâmetros, específico para o transporte, pelo menos, o oxímetro de pulso deve, obrigatoriamente, acompanhar o transporte de todos os pacientes críticos. Sua bateria deve estar carregada [9].

Quanto aos recursos humanos, 29,1\% dos entrevistados consideram que há médicos, enfermeiros e técnicos de enfermagem qualificados. 8,3\% colocam a necessidade de deslocar funcionário.

\footnotetext{
"Os recursos humanos no caso a enfermagem a gente desloca um funcionário do setor onde a gente possa remanejar." (P3)
}

Na opinião de 16,6\% os recursos humanos são suficientes e capacitados, em contrapartida outros $16,6 \%$ consideram estes recursos insuficientes e descompromissados.

"Eu acho que o recurso humano é muito bom... o corpo de enfermagem, os técnicos 
de enfermagem, os médicos são funcionários de muito tempo, conhecem o serviço, conhecem a rotina, e são muito disciplinados, eu acho que em relação a recursos humanos não vejo problema." (P6)

"Faltam recursos humanos, faltam profissionais médicos pra atuar na remoção." (P16)

$\mathrm{Na}$ pergunta "Comente para mim sobre a capacidade dos profissionais que realizam o transporte", $66,6 \%$ consideram os profissionais capacitados, treinados e competentes. $12,5 \%$ relatam que possuem capacidade de improvisar; 8,3\% atribuem a capacidade à educação continuada; $12,5 \%$ associam a capacidade à experiência; $4,1 \%$ dizem que são capazes se aprenderem a fazer; para $12,5 \%$ questiona-se quem transporta e a forma como os pacientes são transportados e 4,1\% dizem que médicos não são treinados para o transporte.

Eu acho que são capacitados, os motoristas, os técnicos, enfermeiras, os médicos... eu acho que eles fazem um bom serviço." (P8)

O transporte inter-hospitalar ocorre com grande frequência e embora seja um procedimento rotineiro, a presença de equipe treinada e disponibilidade de equipamentos adequados para sua realização se associam a menor morbidade para o paciente [1]

$\mathrm{Na}$ opiniáo de $12,5 \%$ dos entrevistados a experiência é fator importante para determinar a capacidade dos profissionais. Eles citam que quem trabalha diretamente no Pronto Socorro são mais capacitados para acompanharem o transporte de pacientes críticos.

\footnotetext{
"A maioria dos profissionais aqui que vão geralmente pra transferência quando é paciente muito crítico são os profissionais do pronto socorro, pois eles já estáo mais acostumados com a urgência, todos são bem capacitados, mas o pessoal que está mais tempo aqui no pronto socorro, geralmente são os que vão para poder dá um suporte melhor para o paciente." (P13)
}

Para outro entrevistado $(4,1 \%)$, a capacidade dos profissionais está relacionada ao conhecimento adquirido. Ressaltando a importância da educação continuada.
"Capacidade é o que a gente aprendeu e se sente capaz de fazer." (P18)

Apesar de a maioria dizer que os profissionais são capacitados, treinados e competentes, um profissional $(4,1 \%)$ referiu que os médicos não são treinados para o transporte.

"Os médicos não são treinados especificamente para fazer transporte" (P3)

$12,5 \%$ dos entrevistados citam que os profissionais que realizam o transporte possuem capacidade de improvisar.

"Eles não só tem que ser um bom profissional, como tem que ter um jogo de cintura, porque às vezes a capacidade da gente não é tanta, então, a gente tem que improvisar." (P12)

Improvisar, segundo o dicionário, significa: fazer, arranjar de repente, sem preparação, organizar as pressas, compor na hora [10]. Em se tratando do transporte inter-hospitalar de pacientes críticos, contar com o improviso é imprudência, negligência e imperícia e, pode colocar em risco a vida do paciente.

É de função de todos os profissionais da saúde, independentemente de grau hierárquico evitar que danos irreparáveis de negligência, imprudência e imperícia venham a acontecer na vida de um indivíduo [11].

\section{Conclusão}

Constata-se que o transporte inter-hospitalar é uma prática cotidiana que merece melhor atenção, visto que os funcionários se mostram interessados em prestar assistência ao paciente e reconhecem a complexidade do tema, admitindo, porém, que lhes faltam recursos humanos e materiais, assim como capacitaçáo, para atuar com segurança e conhecimento. Isto traria benefícios não só para as equipes, que iriam adquirir maior qualidade de atuação, bem como para os pacientes, que é nosso principal objetivo, que receberiam, neste momento, uma assistência de melhor qualidade.

Vale lembrar que os trabalhadores da área da saúde devem estar preparados para atuar durante o transporte de pacientes críticos, visto que é 
uma situação delicada que envolve conhecimento científico, capacidade de tomar decisóes rápidas e capacidade de conviver com o estresse.

Essa reflexão se torna ainda mais relevante quando se pensa que, em sua maioria, os profissionais que atuam no transporte estáo afastados do ambiente hospitalar, e em determinadas situaçóes precisam atuar em condiçóes diferentes das habituais, nem sempre adequadas, por isso é importante que o transporte seja sempre acompanhado por uma equipe multidisciplinar.

\section{Referências}

1. Vieira ALP, Guinsburg R, Santos AMN, Peres CA, Lora MI, Miyoushi MH. Transporte intra-hospitalar de pacientes internados em UTI neonatal: fatores de risco para intercorrências. Rev Paul Pediatr 2007;25(3):2406.

2. Rua F. Oxigenação durante o transporte do doente ventilado. Revista Portuguesa de Medicina Intensiva 1999;8(1).
3. Bardin L. Análise de conteúdo. Lisboa: 2007

4. Lacerda MA, Cruvinel MGC, Silva WV. Transporte de pacientes: intra-hospitalar e inter-hospitalar. In: Sociedade Brasileira de Anestesiologia. Curso de educação à distância em anestesiologia. São Paulo: 2002. p.106-23.

5. Ramos VO, Sanna M C. A inserção da enfermagem no atendimento pré-hospitalar. Rev Bras Enf 2005;58(3):355-60.

6. Conselho Federal de Enfermagem. Resolução Cofen no 379/2011. Brasília: Cofen; 2011.

7. Portal do desenvolvimento. SUS Fácil - Sistema Estadual de Regulaçáo Assistencial. [citado em 2012 Jan 21]. Disponível em: URL: www.portaldodesenvolvimento.com.br

8. Traiber C, Andreolio C, Luchese S. Transporte inter-hospitalar de crianças criticamente doentes. Scientia Médica 2006;16(3):119-25.

9. Pereira Junior GA, Nunes T L, Basile-Filho A. Transporte do paciente crítico. Medicina Ribeirão Preto 2001;34(2):143-53.

10. Houaiss A, Villar MS. Dicionário Houaiss da Lingua Portuguesa. Elaborado no Instituto Antonio Houaiss de Lexicografia e Banco de Dados da Língua Portuguesa S/C Ltda. Rio de Janeiro: Objetiva; 2001.

11. Gomes MJ. Erro Médico. In: Bioética. Brasília: Revista do Conselho Federal de Medicina; 1994. 